\title{
E'ILTERBANK PRECODING FOR MIMO FREQUENCY SELECTIVE CHANNELS: MINIMUM REDUNDANCY AND EQUALIZER DESIGN
}

\author{
Vijaya krishna. A, K. V. S. Hari \\ vkrishna@protocol.ece.iisc.ernet.in, hari@ece.iisc.ernet.in, \\ Dept. of ECE, Indian Institute of Science, Bangalore - 560012.
}

\begin{abstract}
This paper deals with the problem of designing zero forcing finite impulse response (ZF-FIR) equalizers for multiple input multiple output (MIMO) FIR channels, in a polynomial matrix framework. When channel knowledge is available at the transmitter, a precoding operation, which introduces redundancy, can he performed to enable FIR equalization at the receiver. We derive the expression for the minimum redundancy required to render an arbitrary MIMO channel matrix polynomially invertible. The non-uniqueness of the FIR inverse can be used to design equalizers based on different criteria such as noise minimization, low delay and low complexity. We provide a solution for the equalizer which minimizes the output noise power. Simulation results are provided to demonstrate the effectiveness of the proposed design.
\end{abstract}

\section{INTRODUCTION}

Multiple antenna communication systems have attracted a lot of attention due to the substantial increase in data rates that they facilitate. But in the presence of intersymbol interference (ISI), equalization becomes a challenging task. Space-time processing methods [1][2] tackle ISI by converting the frequency selective MIMO channel into a set of parallel flat fading channels. On the other hand, FIR equalizer designs [3] view the FIR channel as a polynomial matrix in the delay operator $z^{-1}$.

Availability of channel knowledge at the transmitter can also be exploited to combat ISI and to improve system performance. Space-time precoding methods [1] use the channel information to allocate power along the singular modes of the block-Sylvester matrix corresponding to the channel. But because of the implicit IR nature of the decoding operation, there is a trade-off between the achievable rate and the performance of the system. In [4], the knowledge of the $M \times N$ channel $H(z)$ is utilized to design a pre-equalizer, j.e, a polynomial matrix $T(z)$ such that $H(z) T(z)=I$, at the transmitter. But this method requires $M \leq N$, and assumes that the channel coefficient matrices are orthogonal.
On the other hand, methods which perform FIR equalization at the receiver [3][5] in general require $M>N$ and the channel matrix to be irreducible. 'A polynomial matrix $H(z)$ is polynomially invertible if and only if it is irreducible, i.e, it has full rank for all $z \neq 0$, including $z=\infty$.

We present a filterbank precoding framework which enables FIR equalization for an arbitrary channel (which may even be singular) of any dimension. At the transmitter, the source signal vector is processed by an FIR prccoder before being transmitted. The precoder is chosen in such a way that the precoded channel is FIR invertible. The minimum redundancy required to accomplish FIR invertibility is derived. It is shown that the design freedom available for the precoder can be used to aid in the design of the noiseminimizing equalizer.

\section{THE PRECODING FRAMEWORK}

Consider the MIMO signal model given by

$$
y(n)=\sum_{k=0}^{L_{I I}-1} H(n-k) x(k)+v(n)
$$

where $x(k)$ is the N-length input vector, $y(n)$ is the $M$ length received signal vector, $H(k), k=0,1, \ldots, L_{H}-1$ is the time domain representation of the $M \mathbf{x} N$ frequency selective MIMO channel, and $v(n)$ is the M-length noise vector. In the absence of noise, we can write

$$
Y(z)=H(z) X(z) \quad \text { । }
$$

in the z-domain. Now, an FIR equalizer, (left inverse) for $H(z)$ exists if and only if $M \geq N$ and $H(z)$ is irreducible. Therefore, given an arbitrary $H(z)$, we wish to find an $N$ x $K(K \leq N)$ precoder matrix $G(z)$, so that the $M \times K$ composite channel matrix $C(z)=H(z) G(z)$ has an FIR left inverse. $N-K$ is the redundancy introduced by the precoder. If the channel matrix $H(z)$ is already FIR invertible, then we can chose $G(z)=I \quad(N=K)$, so that no redundancy is introduced. The question we address here is: what is the maximum $K$ (minimum redundancy $N-K$ ) 
for which $C(z)$ is irreducible?. Before we tackle this question; we will look at some properties of the Smith form of a polynomial matrix.

\subsection{Smith form}

Given a $p \times q$ polynomial matrix $A(z)$ with normal rank $r$, $r \leq \min (p, q)$, we can write [6]

$$
A(z)=E(z) B(z) F(z)
$$

where $E(z)$ and $F(z)$ are unimodular matrices (square irreducible matrices) of sizes $p \times p$ and $q \times q$ respectively. $B(z)$ is a $p \times 4$ matrix of the form

$$
B(z)=\operatorname{diag}\left(\alpha_{1}(z), \alpha_{2}(z), \ldots, \alpha_{r}(z), 0, \ldots 0\right)
$$

such that $\alpha_{i}(z) \mid \alpha_{i+1}(z)$ (i.e, $\alpha_{i}(z)$ divides $\alpha_{i+1}(z)$ ). The monic polynomials $\alpha_{i}(z)$ arc called the invariant polynomials of $A(z)$. We say that $A(z)$ is equivalent to $B(z)$ and write $A(z) \sim B(z)$.

Since $E(z)$ and $F(z)$ are unimodular matrices, it follows from $\mathrm{Eq}$. 3 that $\operatorname{rank}[A(z)]=\operatorname{rank}[B(z)] \forall z$. Therefore, if the minimum rank of $A(z)$ over all values of $z$ is $\tau_{m}$, it can be shown that $\alpha_{1}(z)=\alpha_{2}(z)=\cdots=\alpha_{r_{m}}(z)=1$. If an $n \times k ;(\mathbf{n}>\boldsymbol{k})$ matrix $F(z)$ is irreducible, then its Smith form is

$$
P(z) \sim\left[\begin{array}{c}
I_{k \times k} \\
0_{(n-k) \times k}
\end{array}\right]
$$

The following result is stated in [6].

Theorem 1 Let $P_{i}(z), i=I, 2$, have invariant polynomials $\left\{\alpha_{i 1}(z), \alpha_{i 2}(z), \ldots, \alpha_{i T_{i}}(z)\right\}, i=1,2$, where $r_{i}$ is the normal rank of $P_{i}(z)$. Let $P(z)=P_{1}(z) P_{2}(z)$ have normal rank $r$, with invariant polynomials $\left\{\alpha_{\mathrm{1}}(z),{ }_{,}, \alpha_{r}(z)\right\}$. Then, for $1 \leq k \leq r$ and $i=1,2, \alpha_{i k}(z) \mid \alpha_{k}(z)$.

Proof follows directly from the Binet-Cauchy formula [6].

\subsection{Minimum Redundancy}

Theorem 2 Let $r_{m}$ be the minimum rank of the given $M \times$ $N$ FIR channel $H(z)$. An $N \times K$ precoder $G(z)$ which renders the composite channel matrix $C(z)=H(z) G(z)$ polynomially invertible exists if and only if $K \leq r_{m}$.

Proof:

We will first prove the only if part.

Let $\left\{c_{i}(z)\right\},\left\{h_{i}(z)\right\}$ and $\left\{g_{i}(z)\right\}$ denote the invariant polynomials of $C(z), H(z)$ and $G(z)$ respectively. Suppose $C(z)$ is irreducible. Then

$$
C(z) \sim\left[\begin{array}{c}
I_{K \times K} \\
0_{(M-K) \times K}
\end{array}\right]
$$

We can always choose

$$
G(\ddot{z}) \sim\left[\begin{array}{c}
I_{K \times K} \\
0_{(N-K) \times K}
\end{array}\right]
$$

so that $g_{i}(z) \mid c_{i}(z), 1 \leq i \leq K$.

Since the minimum rank of $H(z)$ is $r_{m}$, we have

$$
h_{1}(z)=\ldots .=h_{r_{n !}}(z)=1
$$

and

$$
h_{r_{m}+1}(z), \ldots . ., h_{r}(z) \neq 1
$$

where $r$ is the normal rank of $H(z)$. Hence, $h_{i}(z) \mid c_{i}(z)$ for $1 \leq i \leq K$ only if $K \leq r_{m}$. Therefore, $C(z)$ is irreducible only if $K \leq r_{m}$.

Now we will prove the if part.

Let $H(z)=U(z) D(z) V(z)$ be the Smith form decomposition of the channel matrix. Choose

$$
G(z)=V^{-1}(z)\left[\begin{array}{c}
I_{K \times K} \\
0(N-K) \times K
\end{array}\right] W(z)
$$

where $W(z)$ is an arbitrary $K \times K$ unimodular matrix. Then, we have

$$
C(z)=H(z) G(z)=U(z) D(z)\left[\begin{array}{c}
I_{K \times K} \\
0_{(N-K) \times K}
\end{array}\right] W(z)
$$

Now, since $r_{m}$ is the minimum rank of $H(z)$, the submatrix formed by the first $r_{m}$ columns of $D(z)$ is

$$
\left[\begin{array}{c}
I_{r_{m} \times r_{m}} \\
0_{\left(M-r_{m}\right) \times r_{m}}
\end{array}\right]
$$

If $K \leq r_{m}$, we have

$$
D^{\prime}(z)=D(z)\left[\begin{array}{c}
I_{K \times K} \\
0_{(N-K) \times K}
\end{array}\right]=\left[\begin{array}{c}
I_{K \times K} \\
0_{(M-K) \times K}
\end{array}\right]
$$

Therefore, $C(z) \sim\left[\begin{array}{c}I_{K \times K} \\ 0_{(M-K) \times K}\end{array}\right]$. Therefore, $C(z)$ is irreducible.

This result can be seen as a generalization to the MIMO case of the minimum redundancy results obtained for the multicarrier modulation case [7].

\subsection{Minimum rank and channel zeros}

Given an $M \times N$ channel $H(z)$ with normal rank $r$, let $\Delta(z)$ denote the g.c.d of the minors of order $r$ of $H(z)$ (if $H(z)$ is square and has full normal rank, then $\Delta(z)$ is its determinant). Expanding $\Delta(z)$ in terms of its factors, we have

$$
\Delta(z)=\left(z-\delta_{1}\right)^{\gamma_{1}}\left(z-\delta_{2}\right)^{\gamma_{2}} \ldots\left(z-\delta_{s}\right)^{\gamma_{s}}
$$


The rank of $H(z)$ drops below the normal rank $r$ only at the channel zeros $\delta_{i}, z=1.2, \ldots, \mathrm{s}$. We now relate the minimum rank of $H(z)$ to the maximum among the multiplicities of the channel zeros.

Corollary 1 Let $r$ and $r_{m}$ be the normal rank and the minimum rank of $H(z)$ respectively. Let $\Delta(z)$ be as in Eq. 5 . Then we have $r_{m} \geq r-\max _{i}\left\{\gamma_{i}\right\}$

A random non-square polynomial matrix is irreducible with probability 1 [8]. Therefore, given an arbitrary $M \times N$ channel $H(z)$ with $\dot{M} \# N$, filterbank precoding utilizes all the degrees of freedom i.e, the throughput is $\min (M, N)$ symbols per channel use. If $M<N$, then the channel can be completely pre-equalized i.e, $H(z) G(z)=I$. If $M>N$, FIR equalization can be accomplished without precoding. Compared to space-time processing methods [1][2], our scheme provides better throughput at a much lesser complexity.

When $M=N$, we need a redundancy of 1 i.e, $K=$ $N-1$. For large $N$, the rate of our scheme is in the same range as that of the space-time precoding method [I].

\section{EQUALIZER DESIGN}

The design of the equalizer can be based on different criteria such as low delay, low complexity and noise minimization. The polynomial matrix framework enables one to utilize many of the results in systems theory literature regarding the construction of inverse systems [9]. In this section, wc derive the noise-minimizing equalizer, using the design freedom available at the precoder.

Given the $M \times K$ irreducible precoded channel $C(z)$, the equalizer design problem is to find a polynomial matrix $F(z)=\sum_{j=0}^{L+1-1} F(i) z^{-i}$ such that $F(z) C(z)=I$. This can also be written as $\mathcal{F C}=\mathcal{3}$, where

$$
\begin{gathered}
\mathcal{F}=\left[\begin{array}{cccc}
F(0) & F(1) & \cdots & F\left(L_{F}-1\right)
\end{array}\right] \\
\left|\begin{array}{cccc}
C(0) & \ldots & C\left(L_{C}-1\right) & \\
& & & \\
& & C(0) & C\left(L_{C}-1\right)
\end{array}\right|
\end{gathered}
$$

and $\mathcal{J}=\left[\begin{array}{llll}I & 0 & \cdots & 0\end{array}\right] \cdot \mathcal{F}$ and $\mathcal{J}$ are $K \times M L_{F}$, and $\mathrm{C}$ is $M L_{F} \times K\left(L_{C}+L_{F}-1\right) . C$ is tall if $L_{F} \geq \frac{K\left(L_{G}-1\right)}{M-K}$. $C$ is full rank if and only if [10]

- $C(z)$ is irreducible and column-reduced.

- $L_{F} \geq \max _{1 \leq j \leq M-K} \nu_{j}^{\frac{L}{-}}-1$, where $\nu_{j}^{\perp}$ are the Kronecker indices of the dual space of $\operatorname{span}\{C(z)\}$.

- The degrees of all the columns of $C(z)$ are equal.

The third restriction can be overcome by deleting the null columns of $\mathrm{C}$ as explained in [3]. Therefore, given an irreducible $C(z)$ and with $L_{F}$ chosen large enough, $\mathrm{C}$ is full rank if $C(z)$ is column-reduced. Any polynomial matrix can be brought to column-feduced form by multiplying it to the right by a suitable unimodular matrix [6]. From the proof of theorem 2. it follows that we have the freedom of choosing a $K \times K$ unimodular factor of $G(z)$. We can choose that unimodular matrix such that $C(z)$ becomes column-reduced, resulting in a full rank $C$. In the following section, superscript $\mathrm{H}$ denotes Hermitian transpose.

Theorem3 If $\mathcal{C}$ is tall and full rank arid the noise $v(n)$ in Eq.I is white, the equalizer which minimizes the output noise power is given by $\mathcal{F}=\mathcal{J}\left(\mathcal{C}^{H} \mathcal{C}\right)^{-1} \mathcal{C}^{H}$.

Proof:

The output of the equalizer can be written as

$$
Y(z)=F(z) C(z) X(z)+F(z) V(z)
$$

Using Eq. 6 and Eq. 7, we can rewrite Eq. 8 as

$$
\mathcal{Y}(n)=\mathcal{F C} \mathcal{X}(n)+\mathcal{F} \mathcal{V}(n)
$$

where $\mathcal{X}^{\prime}(n)$ and $\mathcal{V}(n)$ are the blocked versions of $x(n)$ and $v(n)$, of dimensions $K\left(L_{C}+L_{F}-1\right) \times 1$ and $M L_{F} \times 1$ respectively. The covariance matrix of the noise component of $\mathcal{Y}(n)$ is given by

$$
M y=E\left[\mathcal{F} \mathcal{V} \mathcal{V}^{H} \mathcal{F}^{H}\right]=\mathcal{F} \mathcal{F}^{H}
$$

in view of the white noise assumption. The ooise-minimizing equalizer is obtained by minimizing $\operatorname{tr}\left[\mathcal{F} \mathcal{F}^{H}\right]$ subjecl to the constraint $\mathcal{F} \mathcal{C}=\mathcal{J}$. Using the Lagrange multiplier method, we get

$$
2 \mathcal{F}-\mathcal{L C}^{H}=0
$$

where $\mathcal{L}$ is the $K \times K\left(L_{C}+L_{F}-1\right)$ Lagrange multiplier matrix. From Eq. 11, we have

$$
23 c=\mathcal{L C}^{H} \mathcal{C}
$$

Since $\mathcal{F C}=\mathcal{J}$

$$
\mathcal{L}=2 \mathcal{J}\left(\mathcal{C}^{H} \mathcal{C}\right)^{-1}
$$

From Eq. 11 and Eq. 13, we have

$$
\mathcal{F}=\mathcal{J}\left(\mathcal{C}^{H} \mathcal{C}\right)^{-1} \mathcal{C}^{H}
$$

\section{SIMULATION RESULTS}

Simulations were carried out with $3 \times 2$ channel coefficient matrices $H_{i}$ with independent Gaussian distributed complex coefficients (Rayleigh fading) with an exponential power delay profile. $L_{H}$ was chosen to be $\mathbf{4}$. Source signals were generated from a BPSK constellation. The noise generated 


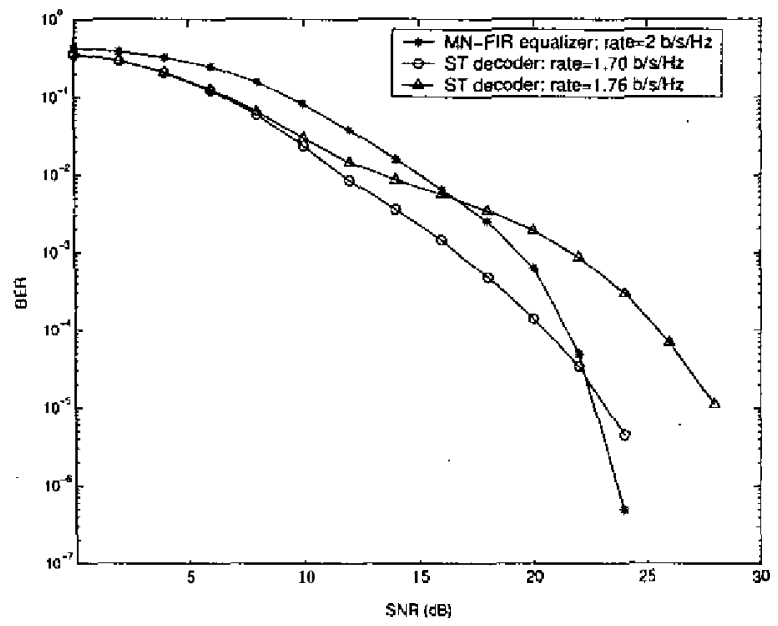

Fig. 1. BER performance of the MN-FIR equalizer (without precoding) and the ST decoder (with precoding).

was spatially and temporally white. The results were averaged over 100 random channels. For comparison purposes, we implemented the space-time (ST) precoding and decoding [1, Lemma 3].

Since $H_{i}$ were randomly generated and $H_{L_{I I-1}}$ was full rank, the channel was both irreducible and column reduced. Hence, noprecoding was performed and the minimum noise FIR (MN-FIR) equalizer was used. ST precoding involved transmission along the eigcnmodes of $\mathcal{H}^{H} \mathcal{H}$ where $\mathcal{H}$ is the block-Sylvester channel matrix. The block size was chosen to be 32 .

- As mentioned in sec.2.2, for non-square random channels, our scheme is full rate, i.e, the throughput for the $3 \times 2$ channel is 2 symbols per channel use ( 2 bits/sec/Hz). In the ST precoding scheme, the rate is limited by the conditioning of $\mathcal{H}^{H} \mathcal{H}$ and by the zero padding used to avoid IBI. Fig. 1 shows the BER curves for the MN-FIR equalizer (with a rate of 2 $\mathrm{b} / \mathrm{s} / \mathrm{Hz}$ ) and the ST decoder (with rates of $1.70 \mathrm{~b} / \mathrm{s} / \mathrm{Hz}$ and $\mathrm{I} .76 \mathrm{~b} / \mathrm{s} / \mathrm{Hz}$ ).

- The ST decoding method involves the eigen decomposition of $\mathcal{H}^{H} \mathcal{H}$, of dimension $N\left(L_{C}+P-1\right) \mathrm{x}$ $N\left(L_{C}+P-1\right)$ with $P \gg L_{C}$. Therefore its computational complexity and latency arc much higher than that of the MN-FIR equalizer. But it has the advantage of trading off rate for performance.

\section{CONCLUSION}

In this paper, we have presented a filterbank precoding scheme for MIMO frequency selective channels. We have derived the minimum redundancy required to enable FIR equalization of an arbitrary channel. We have shown that our scheme achieves better rates than block-based schemes like spacetime precoding, with lesser complexity.

\section{REFERENCES}

[1] A. Scaglione, P. Stoica, S. Barbarossa, G. B, Giannakis, and H. Sampath, "Optimal designs for spacetime linear precoders and decoders," ZEEE Trans. Signal Processing, Vol. 50, pp. 1051-1063, May 2002.

[2] G. G. Raleigh and J. M. Cioffi, "Spatio-temporal coding for wireless communications," IEEE Trans. Cummun., Vol. 46, pp. 357-366, Mar. 1998.

[3] J. K. Tugnait, "FIR inverses to MTMO rational transfer functions with applications to blind equalization," in Proc. 30th Asilomar Conf. on Signals, Systems arid Computers, vol. 1, pp. 295-299, 1997.

[4] H. Sampath, H. Bolcskei, and A. J. Paulraj, "PreEqualization for MIMO wireless channels with delay spread," in Proc. IEEE-VTS Full VTC.2000, vol. 3, pp. I 175-1 I78.2000.

[5] V. Pohl, V. Jungnickel, E. Jorswieck, and C. von Helmolt, "Zero forcing equalizing filter for MIMO channels with intersymbol interference," in Proc. IEEE PIMRC-2002, 2002.

[6] T. Kailath, Linear systems, Englewood Cliffs, NJ: Prenticc-Hall, 1980.

[7] Y.-P. Lin and S.-M. Phoong, "Minimum redundancy for ISI free FIR filterbank transceivers," IEEE Trans. Signal Processing, vol. 50, pp. 842-853, Apr. 2002.

[8] X. -G. Xia, W. Su, and H. Liu, "Filterbank precoders for blind equalization: Polynomial Ambiguity Resistant Precoders (PARP)," IEEE Trans. Circuits arid Systems-l, vol. 48, pp. 193-209, Feb. 2001.

[9] B. Moore and L. Silverman, "A new characterization of feedforward delay-free inverses," IEEE Trans. Info. Theory, vol. 19,pp.126-129, Jan. 1973.

[10] P. Loubaton, E. Moulines, and P. Regalia, "Subspace method for blind identification and deconvolution," in Signal processing advances in wireless communications, Englewood Cliffs, NJ: Prentice-Hall, 2000. 